\title{
Spotted around the web: Researcher job prospects, telehealth, newborn screening
}

\author{
BY JILL ADAMS
}

3 DECEMBER 2021

\section{WEEK OF NOVEMBER $29^{\text {TH }}$}

\section{Research roundup}

- Genetic testing could inform psychiatric drug choice or dosing for many children and adolescents with mental health diagnoses, including autism, according to a population study in Denmark. Pharmacopsychiatry

- Parent reports on the Autism Spectrum Quotient Short Form reveal cultural differences between China and the Netherlands. Journal of Autism and Developmental Disorders

- A survey of authorship in biomedical journals has revealed some journals with a large percentage of papers by prolific authors, who often sit on the editorial boards. PLOS Biology

- Mutations linked to autism are often found in genes important for non-neuronal brain cells, according to an analysis of published transcriptomes. Human Genomics

- Genetic analysis of children with both autism and another neurodevelopmental condition reveals gene variants with roles in regulating gene expression or neuronal communication. Clinica Chimica Acta

- Parents of advanced age may be more likely than younger parents to have a child with autism, according to a birth cohort study in Taiwan. European Child \& Adolescent Psychiatry

- People with 22q11.2 deletion syndrome tend to have a smaller-than-usual cerebellum. Biological Psychiatry

- The prevalence of autism in Wales appears to be 0.51 percent of residents, according to a study of health records. Autism

- Autistic adults and their caregivers have found telehealth visits to be similar to in-person visits, with the added bonus of not having to leave home or interact with other people during the pandemic. Autism

- Mutations in the DSCAM gene, associated with autism and Down syndrome, may alter neuronal development by accelerating dendritic spine maturation. Journal of 


\section{Spectrum | Autism Research News}

https://www.spectrumnews.org

Neuroscience

- An autism-linked variant of the gene ANK2 appears to act through cytoskeletal proteins to limit neuronal branching during development. eLife

- Grapheme-color synesthesia, in which people experience colors when thinking about letters and numbers, seems to correlate to autism traits, especially in people with sensory hypersensitivity. Cortex

\section{Science and society}

- Genetics researchers debate the ethics of sequencing the genomes of all newborn babies. The BMJ

- A collaboration of advocacy groups plans to add chromosome 15 conditions, namely Angelman syndrome, Prader-Willi syndrome and dup15q syndrome, to a newborn screening research program in North Carolina. Spectrum covered newborn screening programs earlier this year. Dup15q Alliance

- The Autistic Self Advocacy Network has developed an equity plan for its organization to better serve its community. Autistic Self Advocacy Network

- The majority of research scientists around the world are pessimistic about their job prospects, according to a survey. Nature

- The American Medical Association employs several autistic people to help with quality assurance testing via a Chicago, Illinois-based employment service. American Medical Association

- President Joe Biden's Build Back Better bill, which was passed by the U.S. House of Representatives 19 November, includes $\$ 150$ billion for home- and community-based services for people with disabilities. Autistic Self Advocacy Network

- A new television series, "As We See It," featuring three autistic roommates played by autistic actors, is slated to premiere 21 January on Amazon Prime Video. Yahoo News

- Research papers should be titled to distinguish whether the focus of study is autism or autism traits, two researchers argue. Autism

- David Mandell is leaving his post as editor-in-chief of the journal Autism. Twitter

- Autism researchers Uta Frith and Francesca Happé have eulogized Michael Rutter. Spectrum remembered the renowned child psychiatrist after his death in October. Nature

- Psychiatrist Sheila Hollins describes her work reviewing U.K.-based cases of people detained in long-term inpatient facilities and her frustration with the government's inaction even the in face of rights violations. The Guardian

- The Australasian Society for Autism Research's virtual conference on autism mental health and suicide prevention is set for 9 December. Australasian Society for Autism Research

- A nonprofit organization in Colorado, where the average time to autism diagnosis is three years, works directly with families to speed the evaluation process. KKTV 11 